\title{
BMJ Open 'Slash and clear' vector control for onchocerciasis elimination and epilepsy prevention: a protocol of a cluster randomised trial in Cameroonian villages
}

\begin{abstract}
Joseph Nelson Siewe Fodjo (D) , ${ }^{1,2}$ Melissa Krizia Vieri, ${ }^{2}$ Leonard Ngarka, ${ }^{1}$ Wepnyu Y Njamnshi, ${ }^{1}$ Leonard N Nfor, ${ }^{1}$ Michel Karngong Mengnjo, ${ }^{1}$ Adam Hendy, ${ }^{3}$ Peter A Enyong, ${ }^{4}$ Dennis Palmer, ${ }^{1}$ Maria-Gloria Basanez, ${ }^{5}$ Robert Colebunders (D) , Alfred K Njamnshi (D) ${ }^{1}$
\end{abstract}

To cite: Siewe Fodjo JN, Vieri MK, Ngarka L, et al. 'Slash and clear' vector control for onchocerciasis elimination and epilepsy prevention: a protocol of a cluster randomised trial in Cameroonian villages. BMJ Open 2021;11:e050341. doi:10.1136/ bmjopen-2021-050341

- Prepublication history for this paper is available online. To view these files, please visit the journal online (http://dx.doi. org/10.1136/bmjopen-2021 050341).

Received 19 February 2021 Accepted 30 July 2021

Check for updates

(c) Author(s) (or their employer(s)) 2021. Re-use permitted under CC BY-NC. No commercial re-use. See rights and permissions. Published by BMJ.

For numbered affiliations see end of article.

Correspondence to

Professor Alfred K Njamnshi; alfred.njamnshi@brainafrica.org

\section{ABSTRACT}

Introduction Onchocerciasis, caused by the filarial Cameroon despite decades of community-directed treatment with ivermectin (CDTI). CDTI is often hampered by coendemicity with loiasis (another filariasis caused by Loa loa) in some areas. Strong epidemiological evidence suggests that $O$. volvulus infection increases the risk for onchocerciasis-associated epilepsy (OAE) among Cameroonian children. This highlights the urgent need to strengthen onchocerciasis elimination programmes in mesoendemic/hyperendemic areas. Novel alternative strategies, such as the 'slash and clear' (S\&C) vector control method, may be required to complement ongoing CDTI to accelerate elimination of transmission. The shortterm impact of S\&C on the biting rates of the blackfly vectors has been demonstrated in other settings. However, its long-term effectiveness and impact on parasitological and serological markers of onchocerciasis transmission as well as on OAE are still unknown.

Methods and analysis We aim to assess the effectiveness of annual S\&C interventions combined with CDTI in reducing onchocerciasis transmission and epilepsy incidence. Eight onchocerciasis-endemic villages located $<5 \mathrm{~km}$ from the Mbam or Sanaga rivers will be randomised to two arms: four villages will receive yearly CDTI only for two consecutive years (Arm 1), while the other four villages will receive CDTI plus annual S\&C for 2 years (Arm 2). Study outcomes (blackfly biting rates, infectivity rates and seroprevalence of onchocerciasis antibodies (0v16 antibodies) in children, prevalence of microfilaridermia and epilepsy incidence) will be monitored prospectively and compared across study arms. We expect that S\&C will have an added benefit over CDTI alone.

Ethics and dissemination The protocol has received ethical approval from the institutional review board of the Cameroon Baptist Convention Health Board (reference number: IRB2021-03) and has been registered with the Pan African Clinical Trials Registry. Findings will be disseminated at national and international levels via meetings and peer-reviewed publications. nematode Onchocerca volvulus, remains endemic in
Strengths and limitations of this study

- This is the first study to investigate the acceptability, cost and sustainability of the slash and clear (S\&C) vector control method in forest zone onchocerciasis foci of Central Africa

- Community-based trial was conducted in eight villages with documented ongoing onchocerciasis transmission and high epilepsy incidence in the Mbam and Sanaga river valleys of Cameroon.

- This is the first study to evaluate the added impact of 'S\&C' on onchocerciasis transmission and epilepsy incidence.

- Study sites may not have comparable baseline characteristics with respect to study outcomes.

- The study follow-up period may be too short to observe any significant difference in epilepsy incidence.

Trial registration number PACTR202101751275357.

\section{BACKGROUND}

Onchocerciasis is a filarial infection caused by Onchocerca volvulus and transmitted between humans via blackfly (Simulium spp) bites. The disease is still endemic in Cameroon, where it is transmitted by blackflies of the Simulium damnosum sensu lato (s.l.) complex, despite long-term community-directed treatment with ivermectin (CDTI). ${ }^{1}$ Coendemicity with loiasis (another filarial infection, caused by Loa loa and transmitted by tabanids) in some areas limits CDTI because ivermectin treatment can lead to severe adverse events in individuals with high $L$. loa microfilarial loads. ${ }^{2}$ In addition to the well-known cutaneous and ocular clinical manifestations of onchocerciasis, there is increasing evidence 
suggesting that infection with $O$. volvulus also affects the central nervous system, resulting in epileptic disorders collectively known as onchocerciasis-associated epilepsy (OAE) ${ }^{34}$ Some of the most robust epidemiological studies supporting this hypothesis were conducted in Cameroon. The findings from two retrospective cohort studies in the Mbam and Lékié divisions in Cameroon revealed increased risks of developing epilepsy following $O$. volvulus infection in childhood, with the risks increasing in a dosedependent manner with increasing microfilarial load. ${ }^{56}$ Moreover, it was recently demonstrated that the intensity of $O$. volvulus infection correlates with $\mathrm{OAE}$ severity and seizure frequency in affected individuals, ${ }^{78}$ further highlighting the need to reduce the burden of onchocerciasis in highly affected foci to prevent poor disease outcomes. Appropriate onchocerciasis elimination measures implemented over a number of years have led to decreasing epilepsy burden in onchocerciasis-endemic foci. ${ }^{9} 10$

In view of the persistent onchocerciasis transmission and difficulties in implementing CDTI in loiasis coendemic areas of Cameroon, it is urgent that complementary strategies are adopted to accelerate elimination of transmission. ${ }^{11}$ It was estimated, via mathematical modelling, that for countries which depend on CDTI only for onchocerciasis control, recent CDTI disruptions brought about by the COVID-19 pandemic would adversely impact onchocerciasis elimination prospects, particularly in places where CDTI is done only once a year as is the case in Cameroon. ${ }^{12}$ The so-called 'slash and clear' (S\&C) method is a community-directed vector control approach with the potential to substantially decrease blackfly population density, biting rates (BR) on humans and thereby onchocerciasis transmission. The method consists of thoroughly and systematically removing the trailing vegetation that acts as substrate for the immature stages (eggs to pupae) of the simuliid vectors in their (fast-flowing) riverine breeding sites. When applied once or twice (according to season) during the year, S\&C can reduce emergence of adult blackflies and consequent BR. Pilot studies in Uganda showed that S\&C can significantly reduce the $\mathrm{BR}$ in endemic foci. ${ }^{13}$ In contrast to $\mathrm{CDTI}$, which entails house-to-house visits by community drug distributors, the S\&C approach fits better in the context of the ongoing COVID-19 health crisis, as it can be implemented by a few community volunteers respecting the physical distancing measures instituted to stall SARS-CoV-2 transmission. In addition, S\&C has been shown to be a cost-effective and environmentally friendly vector control strategy that could accelerate onchocerciasis elimination even in resource-limited settings. ${ }^{14}$

It is expected that a decrease in onchocerciasis transmission would curb the incidence of epilepsy in endemic communities. Indeed, we observed, after several rounds of CDTI in Cameroonian villages, an age shift toward older age groups among persons with epilepsy, suggesting a decreased incidence of epilepsy among younger children. ${ }^{15}$ Similar patterns of reduced epilepsy incidence following onchocerciasis elimination efforts were observed in Uganda, ${ }^{16}$ Nigeria ${ }^{17}$ and West African countries. ${ }^{9}$ These observations stirred the initiation of an interventional study in Maridi (South Sudan), where we introduced biannual CDTI ${ }^{18}$ mapped out blackfly breeding sites and initiated S\&C for vector control in December 2019 to investigate its impact on BR. ${ }^{19}$ However, little is currently known regarding the effects of $\mathrm{S} \& \mathrm{C}$ on serological and parasitological markers of onchocerciasis transmission and disease burden.

\section{Study aim}

We propose a study to investigate the impact of supplementing CDTI with repeated S\&C intervention cycles on blackfly $\mathrm{BR}$ as well as on serological and parasitological markers of onchocerciasis transmission in Cameroonian onchocerciasis-endemic communities. We also intend to assess any changes in the incidence of epilepsy following repeated annual cycles of S\&C.

\section{Study objectives}

The overall objective of this study is to implement the S\&C intervention (as a complementary strategy to CDTI) along rivers near onchocerciasis-endemic villages and assess its potential impact on onchocerciasis and epilepsy. Specifically, we will seek to:

- Map blackfly breeding sites and obtain updated data on onchocerciasis endemicity and ongoing transmission in the Mbam and Sanaga river valleys of Cameroon

- Investigate the acceptability of S\&C in selected Cameroonian villages, which are mesoendemic/ hyperendemic for onchocerciasis

- Evaluate the impact of repeated annual S\&C on transmission patterns and infection trends of onchocerciasis, as well as on epilepsy incidence in the study villages

\section{METHODS \\ Study design}

This will be a prospective, community-based, cluster randomised trial that will last 3 years.

\section{Study setting}

The study will be conducted in eight villages with documented ongoing onchocerciasis transmission and high epilepsy incidence in the Mbam and Sanaga river valleys of Cameroon (figure 1). Potential study villages have been identified during previous field studies ${ }^{1520}$ and recent entomological data are available for some of them ${ }^{21}{ }^{22}$; these are summarised in table 1 . Several members of $S$. damnosum s.l. have been identified breeding along the Mbam river, including Simulium squamosum A, S. squamosum E2 and Simulium mengense, ${ }^{21}$ while $S$. squamosum B has been identified in the Sanaga river. ${ }^{23}$ It is worth noting that a few of the villages listed in table 1 are coendemic with loiasis, which poses the risk of provoking severe adverse effects in some individuals with heavy loiasis load during ivermectin mass drug administration campaigns 


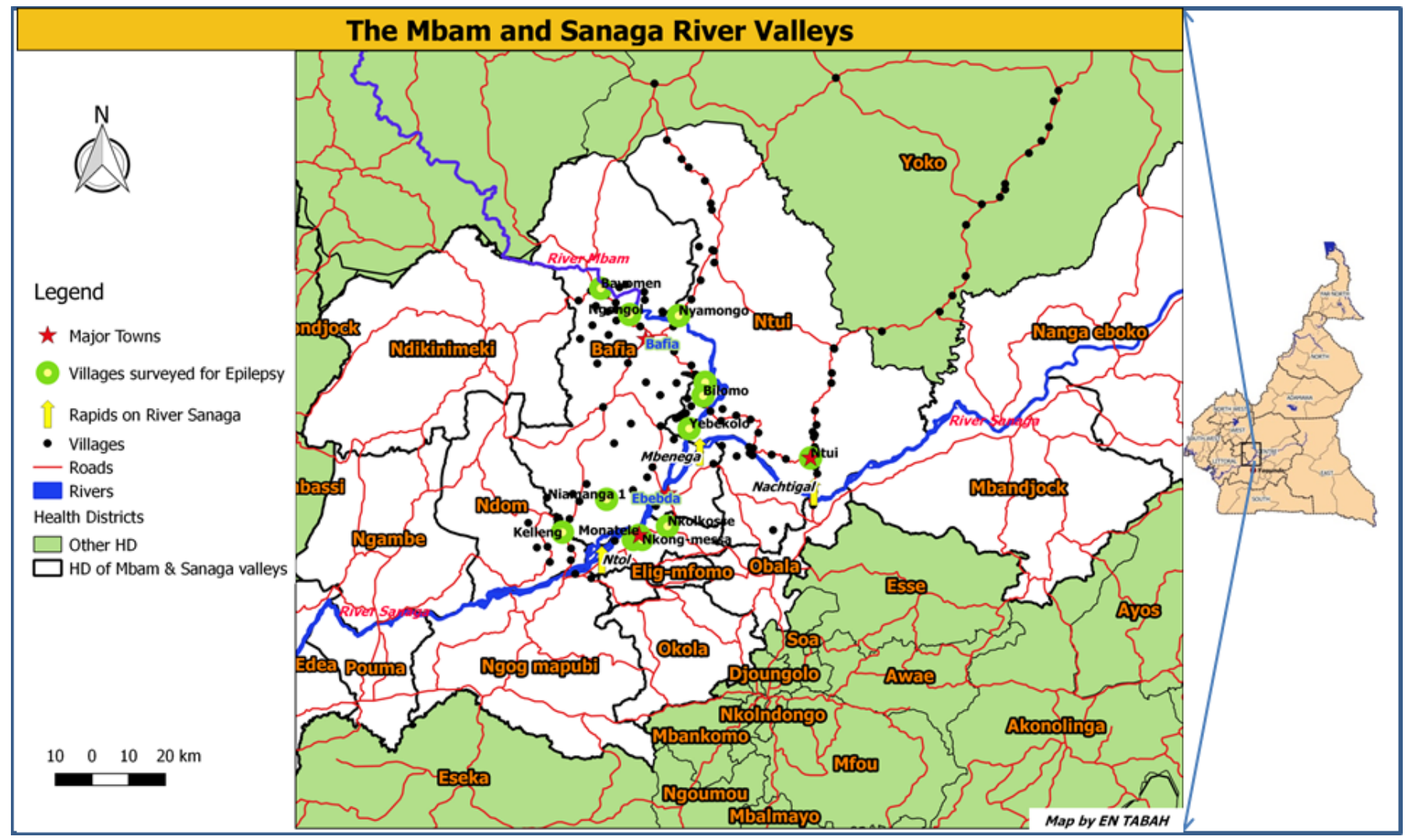

Figure 1 Villages in the Mbam and Sanaga river valleys of Cameroon.

such as CDTI. ${ }^{2}$ The study period is planned from June 2021 to May 2024.

At the start of the project, we intend to map blackfly breeding sites along the portions of the Mbam and Sanaga rivers that flow in the Centre region of Cameroon, and focus on 10-12 potential study villages close to the identified breeding sites for acceptability studies regarding S\&C implementation. Thereafter, eight eligible villages will be selected based on the following inclusion criteria:

- First-line villages (closest to the river banks) located less than $5 \mathrm{~km}$ from the river and with accessible blackfly breeding sites.

- Recent data confirming ongoing onchocerciasis transmission either by parasitological, serological, entomological or clinical (subcutaneous nodules) assessment.

- Acceptability of the S\&C intervention by the local communities.

\section{Study population and samples to be collected}

Our study will enrol different populations and collect serological, parasitological and entomological samples, as described below:

- For Ov16 seroprevalence as a marker for ongoing onchocerciasis transmission: recent data show that antibodies to the $O$. volvulus Ov16 antigen may be detected in children as young as 3 years, ${ }^{19}$ revealing early exposure to the parasite. We propose to obtain blood from children aged 3-6 years to measure Ov16 antibodies as a means to assess very recent onchocerciasis transmission patterns on an annual basis. An advantage of using the 3-6 years age range is that these children are likely to be ivermectin-naïve. ${ }^{24}$

- For the prevalence and intensity of onchocerciasis in study villages: village residents aged 5 years and above will be recruited in each study site to obtain skin snips, which will be used for microscopic detection and enumeration of $O$. volvulus microfilariae following established procedures. ${ }^{25}$ The prevalence and intensity of onchocerciasis infection will be determined annually.

- For the estimation of biting rates and $O$. volvulus transmission rates: adult female blackflies will be captured at each study site by trained village volunteers and both microscopic and DNA-based molecular methods will be used to detect the parasite.

\section{Study procedures}

The proposed research will require the following procedures to be carried out:

- Mapping the blackfly breeding sites: this first step during the project fieldwork will be conducted in a similar manner as in Maridi (South Sudan) in 2019. ${ }^{19}$ River banks will be explored by a team consisting of an experienced entomologist, technicians and local guides; the surfaces of submerged leaves, grasses, sticks and stones will be examined carefully for the presence of blackfly larvae and pupae. Rock surfaces will also be inspected. Larvae and pupae will be placed in a cold box with ice packs or fixed in $70 \%-80 \%$ alcohol for sorting and identification in the laboratory and 
Table 1 Available data from some onchocerciasis-endemic villages in the Mbam and Sanaga river valleys of Cameroon (baseline prevalence and history of CDTI in this area) $)^{1}$

\begin{tabular}{|c|c|c|c|c|c|}
\hline Site & Year & $\begin{array}{l}\text { Ov16 (RDT)* } \\
\text { seroprevalence (\%) } \\
(95 \% \text { Cl)† } \\
\text { (age group in years, } \\
\text { sample size) }\end{array}$ & $\begin{array}{l}\text { Ivermectin } \\
\text { coverage ( } \% \text { of total } \\
\text { population) } \ddagger\end{array}$ & $\begin{array}{l}\text { Mean monthly } \\
\text { biting rate (flies/ } \\
\text { person/month) }\end{array}$ & $\begin{array}{l}\text { Epilepsy } \\
\text { incidence in } \\
\text { village§ }\end{array}$ \\
\hline Bilomoף & 2017 & $\begin{array}{l}46.9(39.0-55.0) \\
(7-10, n=145)^{15}\end{array}$ & $64.1^{15}$ & NA & $227^{15}$ \\
\hline Nyamongof & 2017 & $\begin{array}{l}46.5(37.1-56.2) \\
(7-10, n=101)^{20}\end{array}$ & $61.1^{\star * 47}$ & $19431^{21}$ & $181^{20}$ \\
\hline Ngongolף & 2017 & $\begin{array}{l}42.4(32.4-53.0) \\
(7-10, n=85)^{20}\end{array}$ & $61.1^{\star * 47}$ & NA & $152^{20}$ \\
\hline Bayomenๆ & 2017 & $\begin{array}{l}55.4(46.1-64.2) \\
(7-10, n=112)^{20}\end{array}$ & $61.1^{\star \star 47}$ & $50531^{21}$ & $71^{20}$ \\
\hline Biatsottaף & 2017 & NA & $61.1^{\star * 47}$ & $33390^{22}$ & $500^{5}$ \\
\hline Kelleng†† & 2018 & $\begin{array}{l}52.0(33.5-70.0) \\
(7-10, n=25)^{15}\end{array}$ & $69.2^{15}$ & NA & $98^{15}$ \\
\hline 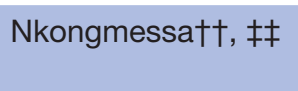 & 2019 & $\begin{array}{l}22.2(12.5-36.3) \\
(6-10, n=45) \S \S\end{array}$ & NA & NA & $2227^{6}$ \\
\hline 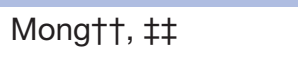 & 2019 & $\begin{array}{l}62.8(47.9-75.6) \\
(6-10, n=43) \S \S\end{array}$ & NA & NA & NAףף \\
\hline
\end{tabular}

${ }^{*}$ Ov16 seroprevalence measured by rapid diagnostic test.

†95\% Cls (Wilson score intervals).

$\ddagger$ Proportion treated over total population aged $\geq 5$ years.

§Incidence per 100000 person-years.

१Mbam river valley.

${ }^{\star \star}$ Overall ivermectin coverage in the Bafia Health District, Mbam valley (2014).

††Sanaga river valley.

$\ddagger \ddagger$ Coendemic with loiasis.

$\S \S$ Unpublished data.

ПП|Anecdotal reports of frequent epilepsy.

CDTI, community-directed treatment with ivermectin; NA, Not available.

some samples will be placed in Carnoy's fixative in the field for later cytotaxonomic studies. ${ }^{26}$ The GPS coordinates will be recorded at each breeding site and will be used to guide the future S\&C intervention. Mapping of breeding sites will be repeated during the last months of the research project (during both dry and rainy seasons) to investigate any changes in the location of blackflies' oviposition sites following the implementation of S\&C.

- Qualitative studies to assess S\&C acceptability (formative research): these will include focus group discussions (FGDs) and in-depth interviews with community leaders/members on S\&C perceptions, all of which will be conducted during the first year of the project. In each village, the research team will recruit between 6 and 8 opinion leaders (chiefs, teachers, religious authorities and quarter heads) for FGDs in order to better understand the extent of the nuisance caused by blackflies as well as their views and acceptability regarding S\&C. Additionally, at least five in-depth interviews will be conducted with other village leaders/members on these same topics. Data from the FGDs and in-depth interviews will be transcribed by trained field staff, verbatim from French/local languages into English. Thereafter, a framework analysis will be conducted, consisting of coding the data and analysing them in six stages: familiarisation, identification of a thematic framework, indexing, charting, mapping and interpretation. ${ }^{27}$

- Selection and randomisation of study sites: villages close to breeding sites $(<5 \mathrm{~km})$, and located in two geographical zones at a reasonable distance from one another $(>15 \mathrm{~km}$ to minimise contamination across study arms), will be considered for inclusion in the study. Although wind-assisted flight ranges of over $400 \mathrm{~km}$ have been documented for $S$. damnosum species, ${ }^{28}$ we chose the $15 \mathrm{~km}$ distance between the study zones since this is more logistically feasible, and it is unlikely that blackflies to travel that far when host seeking if there are closer communities with human blood hosts to feed on. The final selection of the eight study villages (four villages in each zone) will be done based on the aforementioned village inclusion criteria. Thereafter, the investigators will allocate each zone to one study arm by tossing a coin: the intervention arm (comprising of four villages where S\&C will be 
implemented during year 2 and year 3 of the project, in addition to the usual annual CDTI) and the control arm (comprising of four villages where no S\&C will be done, but annual CDTI will be maintained).

- Blackfly catching, processing and biting rate measurements: blackfly catches will be made by trained community volunteers following the human landing catch (HLC) method described by Walsh $e t a l,{ }^{29}$ which is still the gold standard technique recommended by the WHO. At each study village, a blackfly catching site will be established in a relatively open area near the river. A team of two collectors will work alternate hours between 07:00 and 18:00 to catch and record the number of blackflies landing on their feet and legs, exposed below the knee. Homemade aspirators, rather than individual tubes, will be used to make collections since we anticipate encountering very high numbers of biting flies (table 1). Blackfly catches and measurements of BR will be made during 1 full week at baseline, and for 3 consecutive days each month for the remaining duration of the study. The first 12 months of the project will be used to establish baseline entomological data prior to the S\&C intervention. BR will then be monitored during the second and third years when $\mathrm{S} \& \mathrm{C}$ is implemented in the intervention villages. We will offer ivermectin treatment to all volunteers involved in BR assessment using the HLC. During the prospective entomological follow-up, one of the 3 days reserved for BR assessment each month will be used for blackfly dissection to verify parity as previously described. ${ }^{30}$ The dissected blackflies will also be microscopically examined for the presence of $O$. volvulus larvae. The number, developmental stage and location of larvae in the blackflies will be used to aid the calculation of transmission potentials, that is, the number of infective larvae per person per unit time: monthly and annual transmission potentials. Non-dissected blackflies will be placed in $90 \%$ ethanol and stored at $-20^{\circ} \mathrm{C}$ for future molecular analysis.

- Implementation of S\&C in intervention villages: this step will entail:

a. Training community volunteers on the $\mathrm{S} \& \mathrm{C}$ procedures as previously documented, ${ }^{13}$ with emphasis on safety measures for COVID-19 prevention (personal protective equipment and safe interpersonal distances).

b. Clearing the vegetation: the trained volunteers will be deployed to the blackfly breeding sites, where they will cut the trailing vegetation from the water and throw it on the river banks to dry, thereby killing the adherent blackfly eggs, larvae and pupae. ${ }^{13}$ Ideally, S\&C will be done between the months of April and May of the second and third study years because April marks the transition from the dry to the rainy season in Cameroon, which is the recommended period for the intervention. ${ }^{31}$ Given the nature of the S\&C intervention, blinding will not be applied in this study.
- Molecular analysis of the blackflies: real-time PCRbased pool screening will be performed on the stored blackflies as described by Hendy et al. ${ }^{26}$ After discarding blackflies with visibly distended abdomens (which may have fed on the vector collector prior to being captured), the remaining blackflies will have their heads separated from their bodies in a Petri dish using sterilised number 3 entomological pins and a dissecting microscope. Heads and bodies will be pooled separately (up to 100 blackflies per pool); these pools will be analysed using a triplex real-time PCR capable of differentiating $O$. volvulus from Onchocerca ochengi (a bovine parasite, also transmitted by blackflies). The probes, primers and cycling conditions will be similar to those used in a protocol used by Hendy et $a .^{26}$ A positive pool of blackfly 'bodies' (thoraces and abdomens) will be interpreted as being infected with microfilariae acquired during a recent blood meal or developing $O$. volvulus larvae (L1 and L2), whereas a positive pool of 'heads' will be interpreted as containing infective L3 stage ( $\mathrm{L} 3 \mathrm{H})$ parasites. The prevalence of $O$. volvulus infection among blackflies will be estimated using the algorithm described by Katholi $e$ e $a l^{32}$ Mathematically, the prevalence will be given by the formula:

$$
p=1-\left(\frac{k}{m}\right)^{\frac{1}{n}}
$$

where $\mathrm{p}=$ prevalence of infection in blackflies, $\mathrm{m}=$ number of pools of blackflies analysed, $\mathrm{n}=$ number of blackflies per pool, and $\mathrm{k}=$ number of pools that tested negative.

- These procedures will provide important information about the proportion of blackflies that are infected with $O$. volvulus infective larvae and permit an accurate calculation of the infective BR and transmission potentials. Pooled PCR on stored blackflies will be performed twice a year, to ensure that the blackfly samples are not stored for more than 6 months before analysis.

- Collection and processing of dried blood spots (DBS): in each study village, eligible children aged 3-6 years for whom parental consent has been granted will be finger pricked. A few drops of blood will be collected on (Whatman 3) filter papers to prepare DBS, which will be stored in a cooler box with desiccant for future Ov16 ELISA. We will use the recommended horseradish peroxidase (HRP) developed ELISA protocol for measuring Ov16 IgG4 titres in $\mathrm{DBS}^{33}$ : each well on the 96-well ELISA plate will be coated with $100 \mu \mathrm{l}$ of $5 \mu \mathrm{g} / \mathrm{mL}$ Ov16 antigen diluted in phosphatebuffered saline (PBS) and incubated overnight at $4^{\circ} \mathrm{C}$. The DBS will be eluted overnight at $4^{\circ} \mathrm{C}$ in $200 \mu \mathrm{l}$ of PBS, and the eluate transferred in replicates to the sensitised wells of the ELISA plate. After successively washing the plates, adding the HRP-conjugated goat anti-mouse antibody, and stopping the reaction using hydrochloric acid, the plates will be read in a 
spectrophotometer at $450 \mathrm{~nm}$. DBS collection will be done annually during 3 years of the project (in the month of March-April, just before the S\&C period), while the Ov16 ELISA testing will be done in June to minimise DBS storage duration. These procedures will permit us to monitor changes in onchocerciasis transmission in all study sites.

- Skin snip collection: skin snips (one from each iliac crest) will be obtained from each participant aged 5 years and above, resident in the selected villages for microscopic detection and enumeration of $O$. volvulus microfilariae. This will also be performed annually (before the CDTI campaign which takes place every July) to monitor changes in the prevalence and intensity of onchocerciasis in the study sites. Skin snips will be collected with 2-mm Holth corneoscleral punches, incubated in 96-well plates for 24 hours in saline solution, and the medium will be examined microscopically by trained laboratory technicians with experience in onchocerciasis diagnosis for detection and enumeration of microfilariae. ${ }^{25}$ During the examination of the skin snips under a microscope, particular attention will be paid to the morphology and size of the observed microfilariae since $L$. loa microfilariae have been reported to sometimes occur in skin snips. ${ }^{34}$ The mean of the $O$. volvulus microfilarial counts from the two skin snips of each participant will be obtained and expressed as the number of microfilariae per snip. The crude microfilarial prevalence will be calculated as the number of skin-snip-positive individuals (in either or both snips) divided by the total number of individuals examined. The mean intensity of infection in the village will be calculated as the arithmetic mean number of microfilariae/snip across all examined individuals. The community microfilarial load (CMFL) will be calculated as the geometric mean number of microfilariae/snip among those aged $\geq 20$ years. $^{35}$

- Epilepsy surveys: during the early months of the project, community-based epilepsy surveys will be organised in the study sites using a door-to-door approach, which is the gold standard for epilepsy epidemiological studies. ${ }^{36}$ Identification of persons with epilepsy will be done using a two-step approach; first, households will be visited by the research team. After informed consent is obtained, family members will be interviewed using a validated questionnaire. ${ }^{37}$ Second, persons screened as suspected epilepsy cases will be examined by a neurologist or a trainee in neurology to diagnose epilepsy according to the International League Against Epilepsy's definitions. ${ }^{38}$ The homes of confirmed epilepsy cases will be geolocated (to study their geographical distribution around blackfly breeding sites). ${ }^{39}$ A baseline cumulative incidence of epilepsy in each village will be calculated by totalling epilepsy cases with seizure onset within the past 3 years, and dividing by the survey population. ${ }^{15}$ During the last months of our project, we intend to repeat the epilepsy surveys, calculate a final cumulative incidence of epilepsy and compare any changes over time and across study arms. In addition, we will investigate changes in the incidence of epilepsy potentially caused by onchocerciasis. This will be done by identifying cases that meet the following six OAE criteria during the baseline and follow-up surveys: (1) a history of at least two unprovoked epileptic seizures at least 24 hours apart; (2) living at least 3 years in an onchocerciasis-endemic region; (3) living in a village with high epilepsy prevalence and with families with more than one child with epilepsy; (4) no other obvious cause of epilepsy in the patient's history; (5) onset of epilepsy between the ages of 3 years and 18 years and (6) normal psychomotor development before the onset of epilepsy as reported by parents. ${ }^{10}$ Potential 'obvious causes of epilepsy' that will be considered in this study include: a history of a perinatal event, severe malaria, encephalitis or meningitis, and head injury with loss of consciousness all within the 5 years preceding the onset of epileptic seizures.

\section{Sample size calculations}

Given the cluster-randomised design, the sample size calculations take into account the following parameters: two study arms (intervention arm and control arm); four clusters/villages per study arm (ie, eight study villages in total); intracluster correlation coefficient of $0.01^{40}$ and coefficient of variation (estimated difference in cluster size) of 0.1. Sample size calculations were done using the clusterPower package in the software $\mathrm{R}^{41}$

- For Ov16 seroprevalence in children as a marker for recent exposure to onchocerciasis: based on data from South Sudan, ${ }^{19}$ where onchocerciasis transmission levels are comparable with our study villages in Cameroon, we estimate that about $20 \%$ of children aged 3-6 years in the study villages will be Ov16 positive. Assuming a $50 \%$ reduction of Ov16 seropositivity in this age group after 2 years of repeated annual S\&C in the intervention villages, such that the endpoint Ov16 seroprevalence will be $10 \%$ and $20 \%$ in the intervention arm and the control arm respectively, and considering 4 clusters (villages) in each arm, an average cluster size of at least 96 would be required to detect a statistically significant difference with a $5 \%$ precision level and $80 \%$ power. Accordingly, at least $96 \times 4=384$ children should be Ov16 tested in each study arm each year, with the highest possible proportion of 3-year olds.

- For skin snips in participants aged $\geq 5$ years: considering that baseline microfilarial prevalence is about $40 \%$ in many study villages (as recently reported in villages of the Mbam and Sanaga river valleys in Cameroon ${ }^{1}$ where our study sites are located) and assuming that with 2years of annual CDTI the prevalence of positive skin snips decreases by $25 \%,{ }^{12}$ at least 356 individuals ( 89 per village on average) should be skin 
snipped in each arm to detect a significant change in onchocerciasis prevalence. We do not expect that the reduced biting rates due to $\mathrm{S} \& \mathrm{C}$ will translate into reduced microfilarial prevalence during our study period, since previously acquired adult worms would be producing microfilariae even if exposure to new incoming worms were greatly reduced by decreasing vector BR. Therefore, the sample size for skin snipping is not powered to compare changes in onchocerciasis prevalence across arms, but rather for before-after comparisons within each arm. However, we will investigate changes in $O$. volvulus infection intensity and CMFL across the study arms.

\section{Study outcomes and data analysis plan}

The following study outcomes will be of interest:

- Primary outcomes: blackfly biting rates and Ov16 seroprevalence in children, with particular reference to the newly recruited 3-year olds for each year of the study.

- Secondary outcomes: microfilarial prevalence and intensity in study villages, $O$. volvulus transmission potentials and epilepsy incidence

Mean blackfly biting rates will be compared between the study arms at different time points, and also longitudinally using linear mixed models to assess the effects of S\&C over time. Given the limited number of clusters, the between-within method will be applied for small sample corrections. ${ }^{42}$ Besides monitoring the absolute values for BR in study sites, we will also analyse the percentage change in BR every 12 months following the implementation of S\&C, and this will be compared between study arms. Furthermore, the proportion of persons with positive skin snips or children with positive ELISA tests will be compared across the study arms using the $\chi^{2}$ test, and associations with the S\&C intervention will be investigated using clustered logistic regression analyses. Ninetyfive per cent CIs will be calculated for all the metrics recorded.

\section{Data handling and storage}

Collected data will be codified and entered into secure, password-protected spreadsheets. All data files will be centralised and stored in a secured central server. Namelinked information on participants and identification codes will remain confidential and will be accessible to the principal investigators to analyse results by longitudinal follow-up of individuals and to communicate relevant results to participants or communities. Final anonymised datasets will be deposited in appropriate data repositories and available on reasonable request.

\section{Patient and public involvement}

Although participating village residents were not directly involved in the design of this research protocol, our project seeks to address their longstanding complaints about blackfly nuisance. Study findings will be communicated to the village communities as the project advances.

\section{Ethics and dissemination}

This study will be conducted in accordance with good clinical practices, the Declaration of Helsinki, and applicable regulatory requirements. Ethical clearance to conduct the trial has been obtained from the institutional review board (IRB) of the Cameroon Baptist Convention Health Board (reference number: IRB202103). An administrative pre-authorisation for research was obtained from the Regional Delegation of Public Health of the Centre Region (No. 1156/AP/MINSANTE/SG/ DRSPC of 15.02.2021), and research permits will be delivered by the Ministry of Scientific Research and Innovation of Cameroon. Furthermore, before the start of research activities in the villages, the research team will hold meetings with community leaders and healthcare workers of the selected sites to obtain their collaboration. The purpose, aims and procedures of the study will be discussed with regard to the potential risks and benefits for the community. Prior to enrolment of participants into the study, aims and procedures will be explained by the research team in the language of their choice, and signed or thumb-printed informed consent will be obtained from participants, or their parents/caregivers for minors; additionally, assent will be obtained from adolescents (aged 12-20 years). Open communication will be maintained between the researchers and the community throughout the study period. Restitution of relevant research findings will be done via meetings with the community leaders and reports to the IRB and the public health authorities at the end of the study. Absolute confidentiality of study participants and of the collected data will be respected. The study has been registered with the Pan African Clinical Trials Registry. Study findings will be disseminated in national and international fora via meetings and peer-reviewed publications.

\section{DISCUSSION}

The S\&C technique for onchocerciasis control has already been implemented in the Madi mid-North focus of northern Uganda and found to drastically reduce blackfly biting rates for several months following the intervention both in short-term and long-term S\&C trials. ${ }^{1331}$ A similar observation was made following the introduction of S\&C in Maridi, South Sudan (unpublished data). Recently, attempts were made to better understand the geographical coverage and duration of the effect of an S\&C intervention in reducing $\mathrm{BR} .{ }^{31} \mathrm{BR}$ is a crucial entomological determinant of onchocerciasis transmission, but empirical data regarding the added value of S\&C (over CDTI alone) in reducing community transmission/endemicity of onchocerciasis are currently scarce. The proposed project is innovative in that it will make use of additional markers for onchocerciasis transmission (Ov16 seroprevalence in children and microfilarial infection trends in the population), which are complementary to the BR data. We will start by establishing baseline values in the study villages (already included in the annual CDTI scheme); 
thereafter, during the second and third years of the project, we will implement $\mathrm{S} \& \mathrm{C}$ in selected sites to assess its impact on entomological indices, Ov16 seroprevalence and microfilarial infection trends. Besides comparing our findings across the two study arms, data obtained during the pre- and post-S\&C periods will allow us to conduct cross-over analyses in the intervention villages. This will provide insight into the impact of this intervention on human infection with $O$. volvulus.

A second innovation of the proposed project is that S\&C will be implemented for the first time in villages which are coendemic with loiasis. This is particularly relevant since ivermectin use in such settings has proven to be problematic in classical CDTI settings, where ivermectin is distributed by mass drug administration rather than by other, targeted modalities of delivery, requiring the use of alternative onchocerciasis elimination strategies. One such strategy is testing for $L$. loa and excluding from ivermectin treatment those individuals with dangerous microfilaraemia loads. ${ }^{43}$ Should blackfly control by S\&C be found acceptable, efficacious and cost effective in accelerating onchocerciasis elimination in these villages, it could become common practice in coendemic areas. The fact that local communities readily take ownership of the $\mathrm{S} \& \mathrm{C}$ approach ${ }^{31}$ would make it highly sustainable in rural African settings. Crucially, the ovipositing behaviour of the blackflies will be monitored to ascertain any changes in the location and/or nature of their breeding sites along the rivers in the study sites.

A few limitations must be highlighted in the present protocol. The follow-up period of 2 years after the first S\&C intervention may be too short to detect significant differences between the baseline and follow-up Ov16 seroprevalence and microfilarial prevalence, and it may even be more difficult to observe a noticeable change in epilepsy incidence. Indeed, a meta-analysis suggested that about 1-9 years of onchocerciasis control are required to produce a significant reduction in epilepsy prevalence. ${ }^{9}$ However, prevalent cases of epilepsy are expected to change more slowly than incident cases, which will be the focus of this study. Microfilarial prevalence is also unlikely to change drastically due to transmission reductions in the S\&C arm of the study. This is because the prepatent period of $O$. volvulus (the period between acquisition of infection and detectable microfilariae in the skin) is about 2 years. ${ }^{44}$ Regarding the evolution of Ov16 seroprevalence, some children may be recruited more than once during the study period. However, as we will be recruiting a new cohort of 3-year olds each year, this age group will be important in monitoring annual changes in onchocerciasis transmission. Another limitation that arises due to financial and logistical constraints is that we will be unable to perform elaborate laboratory and imaging investigations to identify/confirm the different causes of epilepsy among the participants. Furthermore, neither investigators nor participants will be blinded regarding the villages that will benefit from the S\&C intervention.
Nevertheless, establishing these baseline findings is still of prime importance as this could be the first step towards a more long-term follow-up of S\&C implementation in these communities and its effects on onchocerciasis transmission and epilepsy. The data collected during this project will be used to parameterise and/or validate existing models of blackfly population dynamics and the impact of vector control on such dynamics, ${ }^{45}$ as well as models of onchocerciasis transmission, ${ }^{46}$ taking into account CDTI coverage, duration and S\&C implementation. Such mathematical models will be used to simulate scenarios to investigate the long-term impact of S\&C on $\mathrm{BR}$, transmission potentials, Ov16 seroprevalence in children, onchocerciasis prevalence and intensity, and OAE.

\section{Perspectives}

This study aims to assess the potential impact of S\&C in reducing vector $\mathrm{BR}$ and onchocerciasis transmission in endemic villages, as well as reducing the incidence of epilepsy. Study results may provide evidence that supplementing CDTI programmes with S\&C could accelerate onchocerciasis elimination and prevent new cases of OAE. The results and lessons learnt from this study will be shared with all interested health authorities in Cameroon, the Cameroon Onchocerciasis Elimination Committee, other African countries endemic for onchocerciasis and crucially, with the affected communities participating in the study.

\section{Author affiliations}

${ }^{1}$ Brain Research Africa Initiative (BRAIN) \& Neuroscience Lab, Faculty of Medicine and Biomedical Sciences, The University of Yaounde I, Yaounde, Cameroon

${ }^{2}$ Faculty of Medicine and Health Sciences, Global Health Institute, University of Antwerp, Wilrijk, Belgium

${ }^{3}$ Department of Pathology, The University of Texas Medical Branch, Galveston, Texas, USA

${ }^{4}$ Research Foundation in Tropical Diseases and Environment, Buea, Cameroon ${ }^{5}$ Imperial College London, London, UK

\section{Twitter Robert Colebunders @Colebunders2}

Acknowledgements We thank Tom Lakwo (Ministry of Health, Uganda) for sharing his slash and clear experience with the research team. We are grateful to Dr EN Tabah for designing a map of the research area.

Contributors JNSF, AKN and RC conceived the study. JNSF wrote the initial draft of the manuscript. MKV, AH, PAE, M-GB, RC and AKN provided substantial contributions to the initial draft. All the authors (JNSF, MKV, LN, WYN, LNN, MKM, AH, PAE, DP, $\mathrm{M}-\mathrm{GB}, \mathrm{RC}$ and $\mathrm{AKN}$ ) critically reviewed different versions of the manuscript and approved the final version prior to submission.

Funding This project is part of the second European and Developing Countries Clinical Trials Partnership (EDCTP2) programme supported by the European Union (grant number: TMA2020CDF-3152-SCONE), with additional funding obtained via the Royal Society of Tropical Medicine and Hygiene (2020 small grants session), both awarded to JNSF. MGB acknowledges joint centre funding (grant number: MR/ R015600/1) by the UK Medical Research Council (MRC) and the UK Department for International Development (DFID) under the MRC/DFID concordat agreement, which is also a part of the EDCTP2 programme supported by the European Union. The sponsors had no role in the design and decision to publish this protocol.

Map disclaimer The inclusion of any map (including the depiction of any boundaries therein), or of any geographic or locational reference, does not imply the expression of any opinion whatsoever on the part of BMJ concerning the legal status of any country, territory, jurisdiction or area or of its authorities. Any such expression remains solely that of the relevant source and is not endorsed by BMJ. Maps are provided without any warranty of any kind, either express or implied.

Competing interests None declared. 
Patient and public involvement Patients and/or the public were not involved in the design, or conduct, or reporting, or dissemination plans of this research.

Patient consent for publication Not required.

Provenance and peer review Not commissioned; externally peer reviewed.

Restitution of relevant research findings will be disseminated via open access peer-reviewed journals and anonymised datasets will be made available in public repositories.

Open access This is an open access article distributed in accordance with the Creative Commons Attribution Non Commercial (CC BY-NC 4.0) license, which permits others to distribute, remix, adapt, build upon this work non-commercially, and license their derivative works on different terms, provided the original work is properly cited, appropriate credit is given, any changes made indicated, and the use is non-commercial. See: http://creativecommons.org/licenses/by-nc/4.0/.

\section{ORCID iDs}

Joseph Nelson Siewe Fodjo http://orcid.org/0000-0003-3544-1239

Robert Colebunders http://orcid.org/0000-0002-1919-1340

Alfred K Njamnshi http://orcid.org/0000-0003-0052-1511

\section{REFERENCES}

1 Kamga G-R, Dissak-Delon FN, Nana-Djeunga HC, et al. Still mesoendemic onchocerciasis in two Cameroonian communitydirected treatment with ivermectin projects despite more than 15 years of mass treatment. Parasit Vectors 2016;9:581.

2 Gardon J, Gardon-Wendel N, Demanga-Ngangue, et al. Serious reactions after mass treatment of onchocerciasis with ivermectin in an area endemic for Loa loa infection. Lancet 1997;350:18-22.

3 Pion SDS, Kaiser C, Boutros-Toni F, et al. Epilepsy in onchocerciasis endemic areas: systematic review and meta-analysis of populationbased surveys. PLoS Negl Trop Dis 2009;3:e461.

4 Colebunders R, Njamnshi AK, Menon S, et al. Onchocerca volvulus and epilepsy: a comprehensive review using the Bradford Hill criteria for causation. PLoS Negl Trop Dis 2021;15:e0008965.

5 Chesnais CB, Nana-Djeunga HC, Njamnshi AK, et al. The temporal relationship between onchocerciasis and epilepsy: a populationbased cohort study. Lancet Infect Dis 2018;18:1278-86.

6 Chesnais CB, Bizet C, Campillo JT, et al. A second populationbased cohort study in Cameroon confirms the temporal relationship between onchocerciasis and epilepsy. Open Forum Infect Dis 2020;7:ofaa206.

7 Abd-Elfarag G, Carter JY, Raimon S, et al. Persons with onchocerciasis-associated epilepsy and nodding seizures have a more severe form of epilepsy with more cognitive impairment and higher levels of Onchocerca volvulus infection. Epileptic Disord 2020;22:301-8.

8 Siewe Fodjo JN, Mandro M, Mukendi D, et al. Onchocerciasisassociated epilepsy in the Democratic Republic of Congo: clinical description and relationship with microfilarial density. PLoS Negl Trop Dis 2019;13:e0007300.

9 Siewe Fodjo JN, Remme JHF, Preux P-M, et al. Meta-Analysis of epilepsy prevalence in West Africa and its relationship with onchocerciasis endemicity and control. Int Health 2020;12:192-202.

10 Colebunders R, Siewe Fodjo JN, Hopkins A, et al. From river blindness to river epilepsy: implications for onchocerciasis elimination programmes. PLoS Negl Trop Dis 2019;13:e0007407.

11 Boussinesq M, Fobi G, Kuesel AC. Alternative treatment strategies to accelerate the elimination of onchocerciasis. Int Health 2018;10:i40-8.

12 Hamley JID, Blok DJ, Walker M, et al. What does the COVID-19 pandemic mean for the next decade of onchocerciasis control and elimination? Trans R Soc Trop Med Hyg 2021;115:269-80.

13 Jacob BG, Loum D, Lakwo TL, et al. Community-directed vector control to supplement mass drug distribution for onchocerciasis elimination in the Madi mid-North focus of northern Uganda. PLoS Negl Trop Dis 2018;12:e0006702.

14 Smith ME, Bilal S, Lakwo TL, et al. Accelerating river blindness elimination by supplementing MDA with a vegetation "slash and clear" vector control strategy: a data-driven modeling analysis. Sci Rep 2019:9:15274.

15 Siewe Fodjo JN, Tatah G, Tabah EN, et al. Epidemiology of onchocerciasis-associated epilepsy in the Mbam and Sanaga river valleys of Cameroon: impact of more than 13 years of ivermectin. Infect Dis Poverty 2018;7:114.

16 Gumisiriza N, Mubiru F, Siewe Fodjo JN, et al. Prevalence and incidence of nodding syndrome and other forms of epilepsy in onchocerciasis-endemic areas in northern Uganda after the implementation of onchocerciasis control measures. Infect Dis Poverty 2020;9:12.

17 Siewe JNF, Ukaga CN, Nwazor EO, et al. Low prevalence of epilepsy and onchocerciasis after more than 20 years of ivermectin treatment in the Imo River Basin in Nigeria. Infect Dis Poverty 2019;8:8.

18 Abd-Elfarag G, Logora MY, Carter JY, et al. The effect of bi-annual community-directed treatment with ivermectin on the incidence of epilepsy in onchocerciasis endemic villages in South Sudan: a study protocol. Infect Dis Poverty 2018;7:112.

19 Lakwo TL, Raimon S, Tionga M, et al. The role of the Maridi dam in causing an onchocerciasis-associated epilepsy epidemic in Maridi, South Sudan: an epidemiological, sociological, and entomological study. Pathogens 2020;9:315.

20 Boullé C, Njamnshi AK, Dema F, et al. Impact of 19 years of mass drug administration with ivermectin on epilepsy burden in a hyperendemic onchocerciasis area in Cameroon. Parasit Vectors 2019;12:114.

21 Hendy A. Blackfly ecology and Onchocerca volvulus transmission in three formerly hyperendemic foci in Uganda, Tanzania and Cameroon. [PhD Thesis] 2018; University of Antwerp. Available: https://repository.uantwerpen.be/docman/irua/7a3e67/13135.pdf

22 Abong RA, Amambo GN, Hamid AA, et al. The Mbam drainage system and onchocerciasis transmission post ivermectin mass drug administration (MDA) campaign, Cameroon. PLoS Negl Trop Dis 2021;15:e0008926.

23 Traore-Lamizana M, Somiari S, Mafuyai HB, et al. Sex chromosome variation and cytotaxonomy of the onchocerciasis vector Simulium squamosum in Cameroon and Nigeria. Med Vet Entomol 2001;15:219-23.

24 Siewe Fodjo JN, Mubiru F, Ukaga C, et al. Low ivermectin use among 5- to 6-year-old children: observations from door-to-door surveys in onchocerciasis-endemic regions in Africa. Int Health 2020;12:72-5.

25 Prost A, Prod'hon J. Le diagnostic parasitologique de l'onchocercose: revue critique des méthodes en usage. Médecine Trop 1978;38:519-32.

26 Hendy A, Krüger A, Pfarr K, et al. The blackfly vectors and transmission of Onchocerca volvulus in Mahenge, south eastern Tanzania. Acta Trop 2018;181:50-9.

27 Iliffe S, Wilcock J, Drennan V. Chapter 5: five main stages in framework analysis. NIHR Journals Library, 2015. https://www.ncbi. nlm.nih.gov/books/NBK286110/

28 Post RJ, Onyenwe E, Somiari SAE, et al. A guide to the Simulium damnosum complex (Diptera: Simuliidae) in Nigeria, with a cytotaxonomic key for the identification of the sibling species. Ann Trop Med Parasitol 2011;105:277-97.

29 Walsh JF, Davies JB, Le Berre R, et al. Standardization of criteria for assessing the effect of Simulium control in onchocerciasis control programmes. Trans R Soc Trop Med Hyg 1978;72:675-6.

30 Duke BO. The differential dispersal of nulliparous and parous Simulium damnosum. Tropenmed Parasitol 1975;26:88-97.

31 Jacob B, Loum D, Munu D, et al. Optimization of slash and clear community-directed control of Simulium damnosum sensu stricto in Northern Uganda. Am J Trop Med Hyg;104:1394-403.

32 Katholi CR, Toé L, Merriweather A, et al. Determining the prevalence of Onchocerca volvulus infection in vector populations by polymerase chain reaction screening of pools of black flies. $J$ Infect Dis 1995;172:1414-7.

33 Golden A, Faulx D, Kalnoky M, et al. Analysis of age-dependent trends in Ov16 IgG4 seroprevalence to onchocerciasis. Parasit Vectors 2016;9:338.

34 Niamsi-Emalio $\mathrm{Y}$, Nana-Djeunga HC, Chesnais CB. Unusual localization of blood-borne Loa loa microfilariae in the skin depends on microfilarial density in the blood: Implications for onchocerciasis diagnosis in co-endemic areas. Clin Infect Dis 2021:ciab255.

35 Remme J, Ba O, Dadzie KY, et al. A force-of-infection model for onchocerciasis and its applications in the epidemiological evaluation of the onchocerciasis control programme in the Volta River Basin area. Bull World Health Organ 1986;64:667-81.

36 Bharucha N, Odermatt P, Preux P-M. Methodological difficulties in the conduct of neuroepidemiological studies in low- and middleincome countries. Neuroepidemiology 2014;42:7-15.

37 Diagana M, Preux PM, Tuillas M. Dépistage de l'épilepsie en zones tropicales: validation d'un questionnaire en Mauritanie. Bull Soc Pathol Exot 2006;1990:103-7.

38 Fisher RS, Acevedo C, Arzimanoglou A, et al. ILAE official report: a practical clinical definition of epilepsy. Epilepsia 2014;55:475-82.

39 Boussinesq M, Pion SDS, Ngangue D. Relationship between onchocerciasis and epilepsy: a matched case-control study in the Mbam Valley, Republic of Cameroon. Trans $R$ Soc Trop Med Hyg 2002;96:537-41. 
40 Killip S, Mahfoud Z, Pearce K. What is an intracluster correlation coefficient? crucial concepts for primary care researchers. Ann Fam Med 2004;2:204-8.

41 Kleinman K, Sakrejda A, Moyer J. clusterPower: power calculations for cluster-randomized and cluster-randomized crossover trials, 2021. Available: https://CRAN.R-project.org/package=clusterPower [Accessed 9 Feb 2021].

42 Leyrat C, Morgan KE, Leurent B, et al. Cluster randomized trials with a small number of clusters: which analyses should be used? Int $J$ Epidemiol 2018;47:321-31.

43 Kamgno J, Pion SD, Chesnais CB, et al. A Test-and-Not-Treat strategy for onchocerciasis in Loa loa-Endemic areas. N Engl J Med 2017;377:2044-52.

44 Prost A. [Latency period in onchocerciasis]. Bull World Health Organ 1980;58:923-5.
45 Routledge I, Walker M, Cheke RA, et al. Modelling the impact of larviciding on the population dynamics and biting rates of Simulium damnosum (s.l.): implications for vector control as a complementary strategy for onchocerciasis elimination in Africa. Parasit Vectors 2018;11:316.

46 Hamley JID, Milton P, Walker M, et al. Modelling exposure heterogeneity and density dependence in onchocerciasis using a novel individual-based transmission model, EPIONCHO-IBM: implications for elimination and data needs. PLoS Negl Trop Dis 2019;13:e0007557.

47 Kamga G-R, Dissak-Delon FN, Nana-Djeunga HC, et al. Audit of the community-directed treatment with ivermectin (CDTI) for onchocerciasis and factors associated with adherence in three regions of Cameroon. Parasit Vectors 2018;11:356. 